\title{
Granulocyte and monocyte apheresis therapy for patients with active ulcerative colitis associated with COVID-19: a case report
}

\author{
Miki Koroku' ${ }^{1}$, Teppei Omori ${ }^{1}$, Harutaka Kambayashi ${ }^{1}$, Shun Murasugi ${ }^{1}$, Tomoko Kuriyama ${ }^{1}$, Yuichi Ikarashi ${ }^{1}$, \\ Maria Yonezawa ${ }^{1}$, Ken Arimura ${ }^{2}$, Kazunori Karasawa ${ }^{3}$, Norio Hanafusa ${ }^{4}$, Masatoshi Kawana ${ }^{5}$, Katsutoshi Tokushige ${ }^{1}$ \\ ${ }^{1}$ Institute of Gastroenterology, ${ }^{2}$ Department of Respiratory Medicine, ${ }^{3}$ Division of Nephrology, Department of Medicine, ${ }^{4}$ Department of Blood \\ Purification, and ${ }^{5}$ Primary Care Center, Tokyo Women's Medical University, Tokyo, Japan
}

Coronavirus disease 2019 (COVID-19), caused by infection with severe acute respiratory syndrome coronavirus 2 (SARS$\mathrm{CoV}-2$ ), is now a pandemic. Although several treatment guidelines have been proposed for patients who have both inflammatory bowel disease and COVID-19, immunosuppressive therapy is essentially not recommended, and the treatment options are limited. Even in the COVID-19 pandemic, adjuvant adsorptive granulocyte and monocyte apheresis may safely bring ulcerative colitis (UC) into remission by removing activated myeloid cells without the use of immunosuppressive therapy. Our patient was a 25-year-old Japanese male with UC and COVID-19. This is the first case report of the induction of UC remission with granulocyte and monocyte apheresis treatment for active UC associated with COVID-19. (Intest Res 2022;20:150-155)

Key Words: COVID-19; Granulocyte and monocyte adsorptive apheresis; Ulcerative colitis; Case reports

\section{INTRODUCTION}

Coronavirus disease 2019 (COVID-19), caused by infection with severe acute respiratory syndrome coronavirus 2 (SARS$\mathrm{CoV}-2)$, has become a worldwide pandemic within a short period of time. ${ }^{1}$ Patients with inflammatory bowel disease (IBD) have a risk of contracting SARS-CoV-2 that is similar to the risk among the general population; however, steroids used in the treatment of IBD increase the risk of SARS-CoV-2 infection. ${ }^{2,3}$ Several treatment guidelines have been proposed for patients with IBD and coexisting COVID-19, but the treatment options for active IBD are very limited because immunosuppression therapy is essentially not recommended. ${ }^{2,3}$ Adsorptive granu-

Received November 16, 2020. Revised January 15, 2021.

Accepted January 19, 2021.

Correspondence to Teppei Omori, Institute of Gastroenterology, Tokyo

Women's Medical University, 8-1 Kawada-cho, Shinjuku-ku, Tokyo 162-

8666, Japan. Tel: +81-3-3353-8111, Fax: +81-3-5269-7507, E-mail: omori.

teppei@twmu.ac.jp locyte and monocyte apheresis (GMA) with an Adacolumn ${ }^{\circledR}$ device has been established as an extracorporeal circulating therapy designed to selectively deplete elevated and activated myeloid cells, and it has shown efficacy in patients with ulcerative colitis (UC) and Crohn's disease. ${ }^{4}$ The present case report is the first description of the induction of remission with GMA treatment for a patient with both active UC and COVID-19. The our ethics committee judged that this case report did not need institutional review board approval. However, the study was performed in according with the principle of Declaration of Helsinki and we had got the agreement of this study from the patient. Written informed consent was obtained.

\section{CASE REPORT}

The patient was a 25-year-old male. He was diagnosed 3 months earlier with UC of the total colitis type. He was started on in- 
duction of remission with 3,000 mg of 5-aminosalicylic acid (5-ASA), which improved his symptoms but did not lead to remission. For this reason, the 5-ASA dose was increased to an extreme dose of 4,000 $\mathrm{mg}$ and budesonide foam was started concomitantly, but there was no remission in the patient's symptoms. He developed severe fatigue and a fever of $38^{\circ} \mathrm{C}$ during the course of treatment. After 2 days of no improvement, the patient consulted the designated medical care provider and underwent a nasopharyngeal polymerase chain reaction (PCR) test for SARS-CoV-2 according to Japanese law. A positive PCR test result was returned the following day, and he was admitted to the hospital the next day according to Japan's Infectious Disease Act. He did not have pneumonia on plain chest X-ray or computed tomography, and his C-reactive protein (CRP) level was $13.0 \mathrm{mg} / \mathrm{L}$. His bloody stools and diarrhea continued without worsening.

The patient did not receive any antiviral drugs or antithrombotic therapy after admission, but his general condition was stable and his CRP was reduced to $3.3 \mathrm{mg} / \mathrm{L}$ on the 6th day. The symptoms of COVID-19 improved and the patient was discharged on the 11th day after the onset of illness. However, the bloody stools persisted, and the patient's CRP had increased to $15.1 \mathrm{mg} / \mathrm{L}$ again at discharge. His UC symptoms did not improve markedly despite continued treatment with 5-ASA and the budesonide foam, and he continued to have manifest bloody stools even after discharge.
A colonoscopy was thus performed at an ambulatory endoscopy center with full personal protective equipment for all staff. Endoscopic images showed a Mayo endoscopic score of 3 and a moderate Ulcerative Colitis Endoscopic Index of Severity of 5 . Deep ulcers were observed on the transverse colon (Fig. 1). In contrast-enhanced computed tomography images,

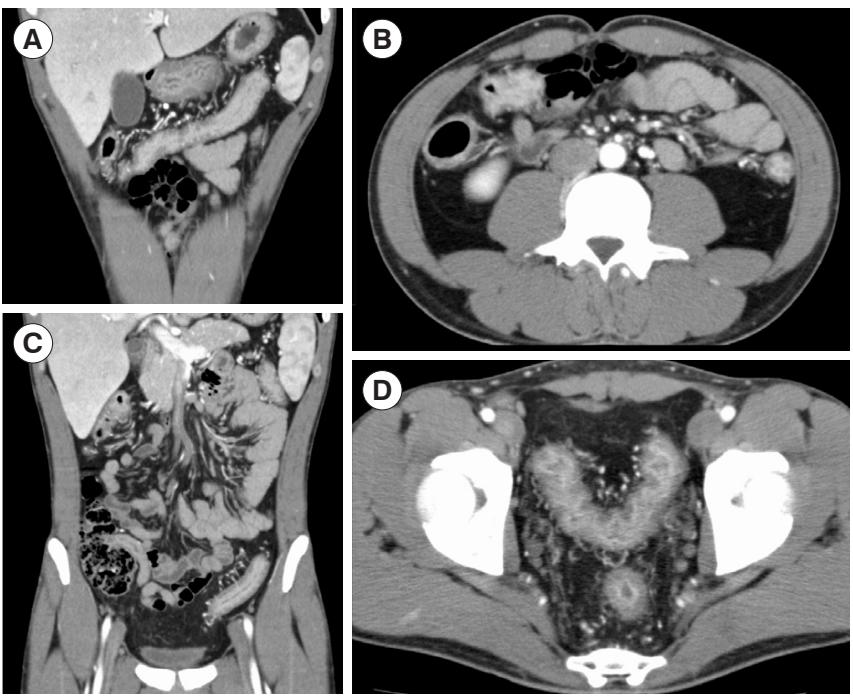

Fig. 2. Contrast-enhanced computed tomography images at the time of admission. Thickening of the intestinal wall was found in continuity from the rectum to the liver curvature. (A) Transverse colon. (B) Liver curvature and ascending colon. (C) Descending colon and sigmoid colon. (D) Sigmoid colon and rectum.
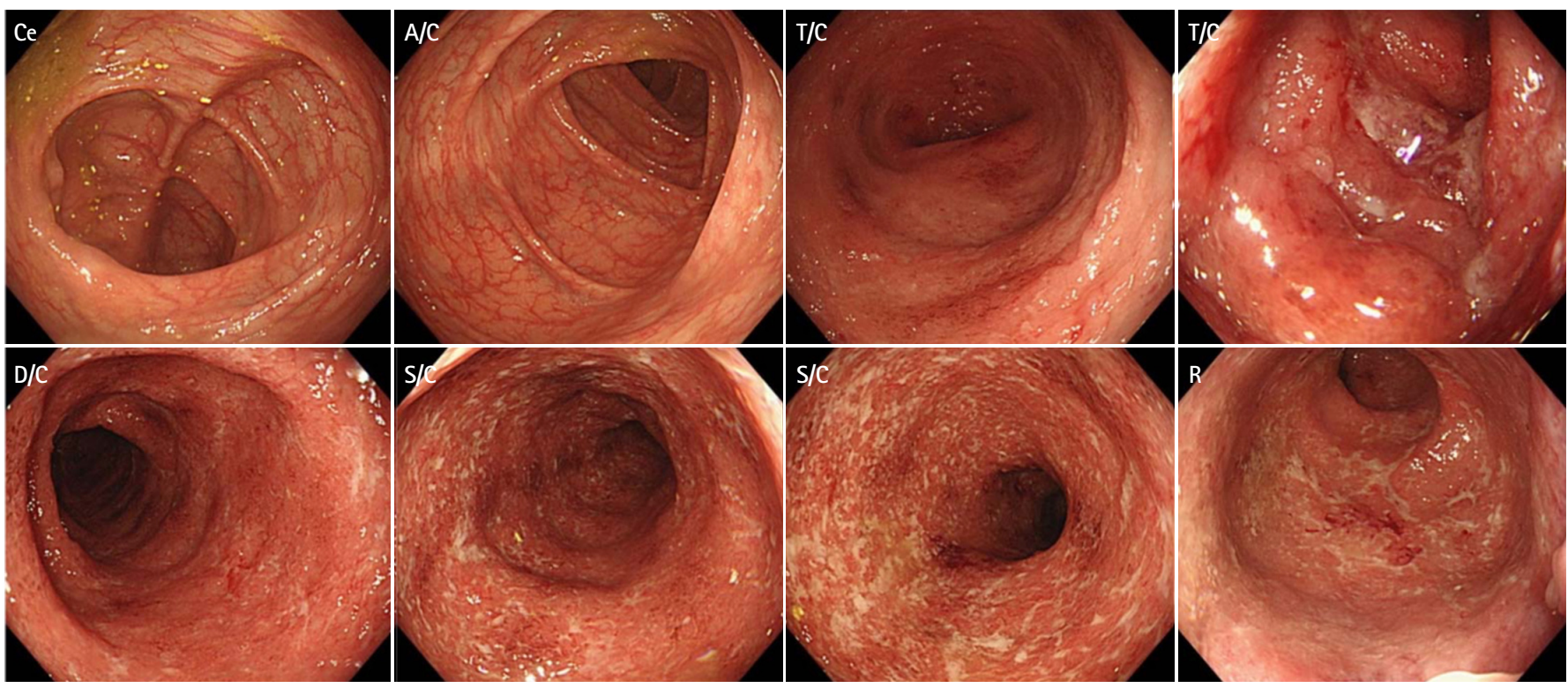

Fig. 1. Endoscopic images on admission. The Mayo endoscopic subscore was 3, and the ulcerative colitis endoscopic index of severity 5 was a moderate endoscopic finding. $\mathrm{Ce}$, cecum; $\mathrm{A} / \mathrm{C}$, ascending colon; $T / \mathrm{C}$, transverse colon; $\mathrm{D} / \mathrm{C}$, descending colon; $\mathrm{S} / \mathrm{C}$, sigmoid colon; $\mathrm{R}$, rectum. 
Table 1. The Patient's Bloodwork Data

\begin{tabular}{|c|c|}
\hline Variable & Value \\
\hline \multicolumn{2}{|l|}{ Blood count } \\
\hline WBC $(/ \mu \mathrm{L})$ & 11,640 \\
\hline $\mathrm{RBC}(/ \mu \mathrm{L})$ & $4.3 \times 10^{6}$ \\
\hline Hemoglobin (g/dL) & 10.6 \\
\hline $\mathrm{PLT}(/ \mu \mathrm{L})$ & $43.1 \times 10^{4}$ \\
\hline \multicolumn{2}{|l|}{ Coagulation fibrinolysis examination } \\
\hline PT (\%) & 93.1 \\
\hline D-dimer $(\mu \mathrm{g} / \mathrm{mL})$ & 0.7 \\
\hline \multicolumn{2}{|l|}{ Biochemistry } \\
\hline $\mathrm{TP}(\mathrm{g} / \mathrm{dL})$ & 6.5 \\
\hline Albumin (g/dL) & 3.0 \\
\hline $\mathrm{CRP}(\mathrm{mg} / \mathrm{L})$ & 26.6 \\
\hline TBIL (mg/dL) & 0.4 \\
\hline AST (U/L) & 13 \\
\hline $\operatorname{ALT}(U / L)$ & 5 \\
\hline $\mathrm{LDH}(\mathrm{U} / \mathrm{L})$ & 230 \\
\hline ALP (U/L) & 189 \\
\hline$\gamma \mathrm{GTP}(\mathrm{U} / \mathrm{L})$ & 12 \\
\hline Amylase (U/L) & 68 \\
\hline $\operatorname{LIP}(\mathrm{U} / \mathrm{L})$ & 25 \\
\hline BUN (mg/dL) & 17.2 \\
\hline $\mathrm{Cr}(\mathrm{mg} / \mathrm{dL})$ & 0.93 \\
\hline $\mathrm{Na}(\mathrm{mEq} / \mathrm{L})$ & 144 \\
\hline $\mathrm{K}(\mathrm{mEq} / \mathrm{L})$ & 4.1 \\
\hline $\mathrm{Cl}(\mathrm{mEq} / \mathrm{L})$ & 106 \\
\hline $\mathrm{Fe}(\mu \mathrm{g} / \mathrm{dL})$ & 15 \\
\hline Ferritin (ng/mL) & 20 \\
\hline $\operatorname{LRG}(\mu \mathrm{g} / \mathrm{mL})$ & 50.20 \\
\hline Fecal immunochemical test (ng/mL) & 9,971 \\
\hline \multicolumn{2}{|l|}{ Infectious disease } \\
\hline $\mathrm{HBs} \mathrm{Ag}$ & Negative \\
\hline $\mathrm{HBc} A b$ & Negative \\
\hline $\mathrm{HCV} A b$ & Negative \\
\hline CMV Ag & Negative \\
\hline Clostridium difficile toxin & Negative \\
\hline SARS-CoV-2 & Positive \\
\hline
\end{tabular}

WBC, white blood count; RBC, red blood count; PLT, platelets; PT, prothrombin; TP, total protein; CRP, C-reactive protein; TBIL, total bilirubin; AST, aspartate aminotransferase; $A L T$, alanine aminotransferase; $L D H$, lactate dehydrogenase; ALP, alkaline phosphatase; $\gamma \mathrm{GTP}$, gamma-glutamyl transpeptidase; LIP, lipase; BUN, blood urea nitrogen; $\mathrm{Cr}$, creatinine; Na, sodium; $\mathrm{K}$, potassium; $\mathrm{Cl}$, chloride; LRG, leucine-rich alpha2 glycoprotein; $\mathrm{HB}$, hepatitis B virus; HCV, hepatitis C virus; CMV, cytomegalovirus; SARS-CoV-2, severe acute respiratory syndrome coronavirus 2 . thickening of the intestinal wall was seen in continuity from the rectum to the liver curvature (Fig. 2). The treatment guidelines of the Japanese Ministry of Health, Labour and Welfare recommended systematic steroids; however, the patient's nasopharyngeal PCR test for SARS-CoV-2 was positive at that time again, and immunosuppression therapy was thus considered to pose a high risk. Although his clinical symptoms were severe (Lichtiger index 16), his endoscopic images were moderate. We therefore planned to induce remission with GMA using an Adacolumn ${ }^{\circledR}$, and we began inpatient treatment with the patient in an infectious disease isolation ward.

The biomarker levels on admission were CRP $26.6 \mathrm{mg} / \mathrm{L}$, leucine-rich alpha2 glycoprotein (LRG) $50.20 \mu \mathrm{g} / \mathrm{mL}$, and fecal immunochemical test (FIT) 9,971 ng/mL (Table 1). We managed the patient with fasting and continued the administration of 5-ASA (4,000 mg) but stopped the budesonide foam, and a total of 5 planned intensive GMA treatments were administered over a 10-day period. At the end of the 5 GMAs, the patient's clinical symptoms had improved to Lichtiger index 3 , and we judged that the patient had achieved remission. We then resumed the budesonide foam and the patient began eating, having completed a total of 10 sessions of GMA therapy; he was then discharged from the hospital. At his discharge, the patient had a Lichtiger index of 5 due to a slight increase in the frequency of bowel movements as a result of the restart of eating, and his biomarkers had improved to CRP $3.3 \mathrm{mg} / \mathrm{L}$, LRG $25.3 \mu \mathrm{g} / \mathrm{mL}$, and FIT $353 \mathrm{ng} / \mathrm{mL}$ (Fig. 3).

\section{DISCUSSION}

The current available evidence indicates that IBD is not a risk factor for COVID-19. ${ }^{3}$ For the treatment of patients with UC that relapses with moderate or worse symptoms despite the use of 5-ASA, systemic steroid therapy is usually the first choice, ${ }^{5,6}$ but the treatment choices for a patient with both active IBD and COVID-19 are limited because immunosuppression therapy is not recommended in principle. ${ }^{2,3}$ Especially in patients with IBD, steroids have been noted to pose a risk of worsening concomitant COVID-19. ${ }^{7}$ GMA is adsorbed by activated myeloid cells into the extracorporeal circulation by an Adacol$\mathrm{umn}^{\circledR}$ filled with cellulose acetate beads. This significantly reduces the inflammatory cytokines interleukin (IL)-1 $\beta$, IL-6, and tumor necrosis factor- $\alpha$ (TNF- $\alpha$ ) in patients with IBD and is a treatment for the induction of remission in $\mathrm{UC}^{8}{ }^{8}$

Japan's national healthcare insurance covers GMA as a treatment for patients with moderate to severe UC. ${ }^{7}$ However, blind- 


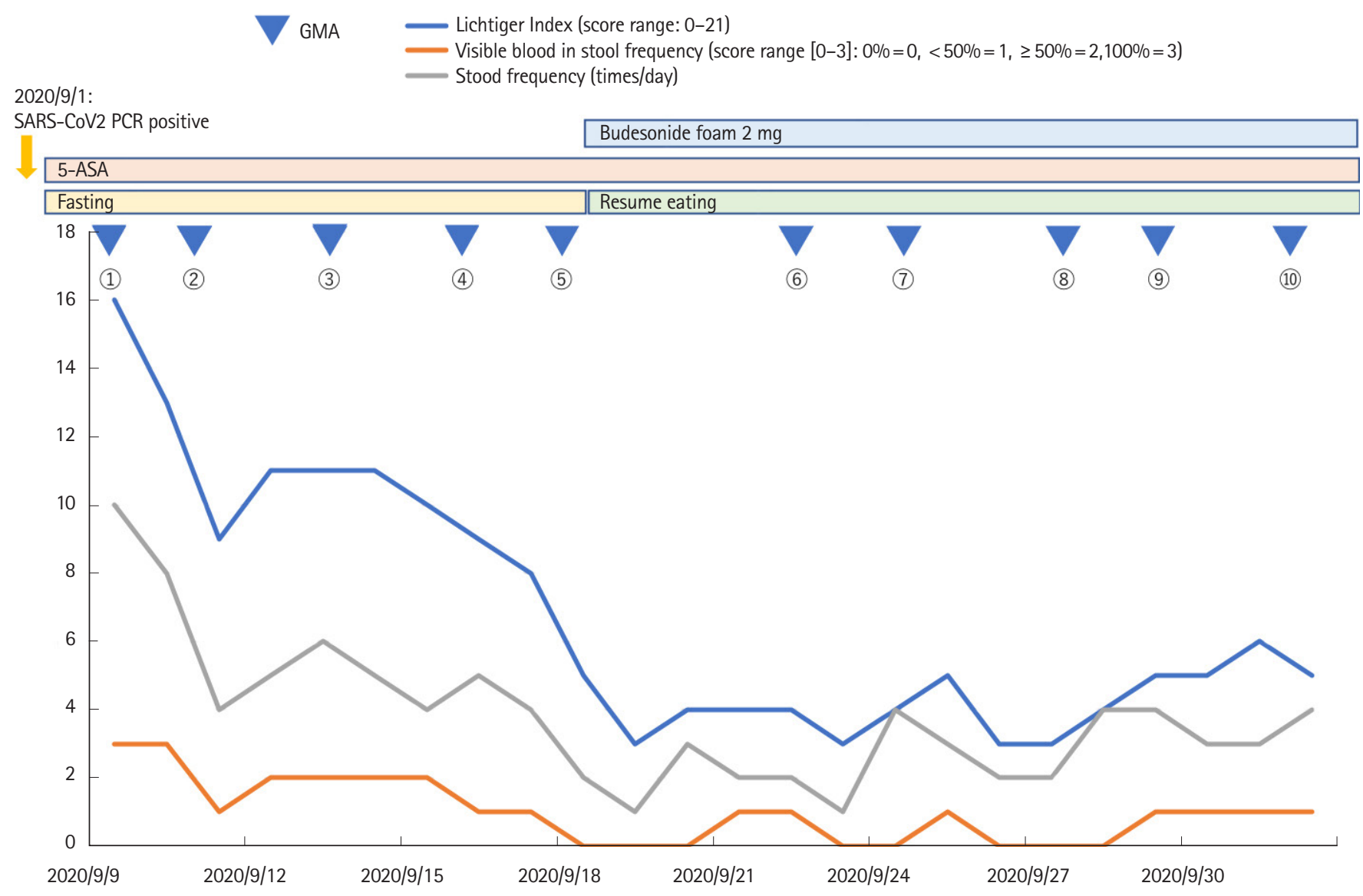

Fig. 3. Clinical course. At the time of the first 5 sessions of granulocyte and monocyte apheresis (GMA), the patient's clinical activity level improved markedly. To bring the patient into further remission, 5 additional GMA sessions were administered, and budesonide foam and eating were resumed. SARS-CoV2, severe acute respiratory syndrome coronavirus 2; PCR, polymerase chain reaction; 5-ASA, 5-aminosalicylic acid.

ed randomized controlled trials conducted in Europe and the United States that used sham columns indicated that GMA did not have a significant therapeutic effect on remission induction. ${ }^{9}$ On the other hand, a meta-analysis examining the efficacy of cytapheresis on remission induction in patients with moderate to severe UC showed that the cytapheresis was better tolerated and safer than conventional drug therapy and may be effective in improving response and remission rates and in tapering corticosteroid doses. ${ }^{10}$ GMA has also been reported to provide a higher rate of remission induction in steroid-naïve patients. ${ }^{11}$ In addition, intensive GMA, in which the GMA is repeated for shorter periods of time (i.e., $2 \times /$ week rather than $1 \times /$ week), has been found to have a higher induction of remission. ${ }^{12}$

Another meta-analysis revealed that intensive GMA is a safe and effective treatment with higher clinical remission and response rates compared to corticosteroids. ${ }^{13}$ Serious infections are not potential adverse events of GMA therapy, and GMA may therefore safely lead to the remission of UC as a treatment for patients with active UC even in the COVID-19 epidemic. However, there are currently no guidelines describing GMA for the treatment of UC in the COVID-19 epidemic.

Our patient presented with COVID-19 in the midst of a UC exacerbation, with no obvious pneumonia complications on imaging and no continuous increased fever. His COVID-19 was thus judged to be a mild case, and the patient had a relatively good course. However, his UC symptom exacerbated and further treatment for UC was required, but the nasopharyngeal PCR test was still positive for SARS-CoV-2. Clinicians need to be careful about the possibility of COVID-19 infection triggering an exacerbation of UC symptoms. He was steroidnaïve; his UC relapses were moderate to severe (the endoscopic images were moderate and the clinical symptoms were severe in his Lichtiger index 16) and a good indication for GMA. The Lichtiger index was developed to evaluate the efficacy and safety of continuous intravenous cyclosporine therapy for 
severe UC and can assess short-term changes in the condition of an individual with UC. ${ }^{14}$

At the patient's endoscopic examination, the staff's full personal protective equipment was confirmed to block SARS$\mathrm{CoV}-2$ infection, but an endoscopic evaluation is difficult to perform frequently for a patient under the diagnosis of COVID-19, ${ }^{15}$ and for this reason, we could not perform a repeat endoscopic examination for our patient at the end of his 10 sessions of GMA. However, the data of CRP and LRG, a new biomarker, ${ }^{16}$ showed a trend toward improvement, as did the patient's clinical symptoms. LRG is a 50-kDa glycoprotein containing 8 domains called leucine-rich repeats that are produced locally in inflammation. LRG is upregulated by IL-6 and other proinflammatory cytokines such as IL-1 $\beta$, IL-22, TNF- $\alpha$, and interferon- $\gamma$. Therefore, LRG reflects intestinal inflammation in UC more sensitively than CRP. ${ }^{16}$

The mechanism underlying the severity of COVID-19 disease has been indicated to be the overproduction of pro-inflammatory cytokines such as IL-1 $\beta$, IL-6, and TNF- $\alpha$ caused by SARS-CoV-2 infection, and this "cytokine storm" causes disseminated intravascular coagulation, which promotes multiple organ failure and lethality. ${ }^{17} \mathrm{COVID}-19$ pneumonia is characterized by an exaggerated immune response (the cytokine storm) with high levels of TNF- $\alpha$ as well as other cytokines. ${ }^{18}$ In other words, remission induction therapy with biologics such as TNF- $\alpha$ antibody drugs for flares of UC complicated by severe COVID-19 may lead to the remission of the UC without exacerbating the pathogenesis of COVID-19, but this is still not fully understood. ${ }^{19}$

In addition, it has been suggested that the removal of activated myeloid cells by GMA may improve not only the pathogenesis of UC but also the pathogenesis of COVID-19 without the need for immunosuppression therapy. ${ }^{20}$ GMA therapy may thus provide a positive outcome for flares of UC complicated by COVID-19, both in the induction of UC remission and the control of COVID-19. In conclusion, our patient's case indicates that GMA for a patient with both active UC and COVID-19 is a safe treatment option for active UC that could lead other patients in this condition to remission.

\section{ADDITIONAL INFORMATION}

\section{Funding Source}

The authors received no financial support for the research, authorship, and/or publication of this article.

\section{Conflict of Interest}

No potential conflict of interest relevant to this article was reported.

\section{Author Contribution}

Study concept and design: Koroku M, Omori T. Data acquisition: Koroku M, Omori T, Kambayashi H, Murasugi S, Ikarashi Y, Arimura K, Kuriyama T, Karasawa K, Yonezawa M. Drafting of the first version of the manuscript: Koroku M, Omori T. Critical revision: Koroku M, Omori T, Hanafusa N, Kawana M, Tokushige K. Approval of the final version of the manuscript: all authors.

\section{ORCID}

Koroku M

Omori T

Kambayashi H

Murasugi S

Kuriyama T

Ikarashi Y

Yonezawa M

Arimura K

Karasawa K

Hanafusa N

Kawana M

Tokushige K

https://orcid.org/0000-0001-7572-1185 https://orcid.org/0000-0002-9959-5168 https://orcid.org/0000-0003-1200-7798 https://orcid.org/0000-0003-1204-1806 https://orcid.org/0000-0002-5201-3618 https://orcid.org/0000-0003-3381-3238 https://orcid.org/0000-0002-6692-4236 https://orcid.org/0000-0002-7934-533X https://orcid.org/0000-0003-2822-6928 https://orcid.org/0000-0002-0563-3320 https://orcid.org/0000-0003-0882-7347 https://orcid.org/0000-0002-1004-7369

\section{REFERENCES}

1. Zhou P, Yang XL, Wang XG, et al. A pneumonia outbreak associated with a new coronavirus of probable bat origin. Nature 2020;579:270-273.

2. Nakase H, Matsumoto T, Matsuura M, et al. Expert opinions on the current therapeutic management of inflammatory bowel disease during the COVID-19 pandemic: Japan IBD COVID-19 Taskforce, Intractable Diseases, the Health and Labor Sciences Research. Digestion 2021;102:814-822.

3. Magro F, Rahier JF, Abreu C, et al. Inflammatory bowel disease management during the COVID-19 outbreak: the ten do's and don'ts from the ECCO-COVID taskforce. J Crohns Colitis 2020; 14:S798-S806.

4. Saniabadi AR, Hanai H, Takeuchi K, et al. Adacolumn, an adsorptive carrier based granulocyte and monocyte apheresis device for the treatment of inflammatory and refractory diseases associated with leukocytes. Ther Apher Dial 2003;7:4859. 
5. Harbord M, Eliakim R, Bettenworth D, et al. Third European evidence-based consensus on diagnosis and management of ulcerative colitis. Part 2: current management. J Crohns Colitis 2017;11:769-784.

6. Matsuoka K, Kobayashi T, Ueno F, et al. Evidence-based clinical practice guidelines for inflammatory bowel disease. J Gastroenterol 2018;53:305-353.

7. Kappelman MD, Brenner EJ, Colombel JF, et al. SECURE-IBD Database Public Data Update [Internet]. c2020 [cited 2020 Nov 7]. https://covidibd.org/current-data.

8. Kashiwagi N, Sugimura K, Koiwai H, et al. Immunomodulatory effects of granulocyte and monocyte adsorption apheresis as a treatment for patients with ulcerative colitis. Dig Dis Sci 2002;47:1334-1341.

9. Sands BE, Sandborn WJ, Feagan B, et al. A randomized, double-blind, sham-controlled study of granulocyte/monocyte apheresis for active ulcerative colitis. Gastroenterology 2008; 135:400-409.

10. Zhu M, Xu X, Nie F, Tong J, Xiao S, Ran Z. The efficacy and safety of selective leukocytapheresis in the treatment of ulcerative colitis: a meta-analysis. Int J Colorectal Dis 2011;26:999-1007.

11. Yamamoto T, Iida T, Ikeya K, et al. A multicenter retrospective study aiming to identify patients who respond well to adsorptive granulomonocytapheresis in moderately to severely active ulcerative colitis. Clin Transl Gastroenterol 2018;9:170.

12. Sakuraba A, Motoya S, Watanabe K, et al. An open-label prospective randomized multicenter study shows very rapid remission of ulcerative colitis by intensive granulocyte and monocyte adsorptive apheresis as compared with routine weekly treatment. Am J Gastroenterol 2009;104:2990-2995.

13. Yoshino T, Nakase H, Minami N, et al. Efficacy and safety of granulocyte and monocyte adsorption apheresis for ulcerative colitis: a meta-analysis. Dig Liver Dis 2014;46:219-226.

14. Lichtiger S, Present DH, Kornbluth A, et al. Cyclosporine in severe ulcerative colitis refractory to steroid therapy. N Engl J Med 1994;330:1841-1845.

15. Ng SC, Mak JWY, Hitz L, Chowers Y, Bernstein CN, Silverberg MS. COVID-19 pandemic: which IBD patients need to be scoped-who gets scoped now, who can wait, and how to resume to normal. J Crohns Colitis 2020;14:S791-S797.

16. Shinzaki S, Matsuoka K, Iijima H, et al. Leucine-rich alpha-2 glycoprotein is a serum biomarker of mucosal healing in ulcerative colitis. J Crohns Colitis 2017;11:84-91.

17. Jose RJ, Manuel A. COVID-19 cytokine storm: the interplay between inflammation and coagulation. Lancet Respir Med 2020;8:e46-e47.

18. Huang C, Wang Y, Li X, et al. Clinical features of patients infected with 2019 novel coronavirus in Wuhan, China. Lancet 2020;395:497-506.

19. Okeke F, Mone A, Swaminath A. The course of SARS-COV2 infection was not severe in a Crohn's patient who administered maintenance anti-TNF therapy overlapping the early pre-symptomatic period of infection. Antibodies (Basel) 2020; 9:42.

20. Kanekura T, Kawahara K. Adsorptive granulocyte and monocyte apheresis: a potentially relevant therapeutic option for COVID-19. Int J Infect Dis 2020;99:1-2. 